\title{
NONSMOOTHING OF ALGEBRAIC CYCLES ON GRASSMANN VARIETIES ${ }^{1}$
}

\author{
BY ROBIN HARTSHORNE, ELMER REES AND EMERY THOMAS
}

Communicated by S. S. Chern, February 20, 1974

1. Introduction. By a cycle $Z$ of dimension $r$ on a nonsingular algebraic variety $X$, we mean a formal linear combination $Z=\sum n_{i} Y_{i}$ of irreducible subvarieties $Y_{i}$ of dimension $r$, with integer coefficients $n_{i}$. The smoothing problem for cycles asks whether a given cycle $Z$ is equivalent (for a suitable equivalence relation of cycles, such as rational equivalence or algebraic equivalence) to a cycle $Z^{\prime}=\sum n_{i}^{\prime} Y_{i}^{\prime}$, where the subvarieties $Y_{i}^{\prime}$ are all nonsingular. Let $X$ be a nonsingular projective variety of dimension $n$ over $\boldsymbol{C}$. Then for each cycle $Z$ of dimension $r$ on $X$ we can assign a cohomology class $\delta(Z) \in H^{2 n-2 r}(X, Z)$. We say that two cycles $Z, Z^{\prime}$ are homologically equivalent if $\delta(Z)=\delta\left(Z^{\prime}\right)$. Our main result is that there are cycles on certain Grassmann varieties which cannot be smoothed, even for homological equivalence, which is weaker than rational or algebraic equivalence.

The smoothing problem was suggested by Borel and Haefliger [2, p. 497] in connection with their study of the cohomology class associated to a cycle. Hironaka [7] showed in characteristic zero that cycles of dimension $\leqq \min \left(3, \frac{1}{2}(n-1)\right)$ can always be smoothed. On a nonsingular variety of any characteristic he showed that if $\operatorname{dim} Z \leqq \frac{1}{2}(n-1)$, then some multiple of $Z$ can be smoothed. Kleiman [8] strengthened the latter result by showing that if $\operatorname{dim} Z<\frac{1}{2}(n+2)$, then $((q-1) !) Z$ can be smoothed, where $q=n-r$ is the codimension of $Z$. The specific cycle which we show to be nonsmoothable was suggested by Kleiman and Landolfi [9], who conjectured that it could not be smoothed.

Thom, in his famous paper [11], studied the closely related question of which homology classes on a smooth manifold can be represented as the homology class of a submanifold. He also answered negatively a question of Steenrod, which asked if every homology class on a manifold was the image by some continuous map of the fundamental class of another manifold. Note that in algebraic geometry if a homology class is the class of some cycle on a nonsingular variety $X$, then it is the image of the

AMS (MOS) subject classifications (1970). Primary 14C10, 14C30, 55G10; Secondary $14 \mathrm{M} 15,55 \mathrm{~F} 40$.

Key words and phrases. Smoothing cycles, Thom space, Steenrod squares.

${ }^{1}$ Research supported by grants from the National Science Foundation. 
fundamental class of a (not necessarily connected) nonsingular variety $Y$ because of Hironaka's theorem on resolution of singularities [6].

Our idea is to apply Thom's method to cycles on the complex Grassmann varieties. We obtain thereby a stronger result, namely that the given cohomology class is not a linear combination of cohomology classes of smooth submanifolds. On the other hand, Thom proves that some multiple of any given cohomology class can be represented as the class of a submanifold. Hence this method cannot answer the remaining open question whether or not some multiple of the cycle in our example can be smoothed.

2. The example. We denote by $P_{n}$ the complex projective $n$-space, $n \geqq 1$, and by $G(m, n), m, n \geqq 1$, the Grassmann variety of complex $m$-planes in a complex $(m+n)$-space. As a complex manifold, $G(m, n)$ has dimension $m n$. Let $\gamma_{m}$ denote the canonical $m$-plane bundle over $G(m, n)$, and let $\eta_{n}$ denote the complementary $n$-plane bundle. Then $\gamma_{m} \oplus \eta_{n}$ is the trivial bundle and hence

$$
c\left(\gamma_{m}\right) \cdot c\left(\eta_{n}\right)=1 \text {, }
$$

where $c$ denotes the total chern class. This equation allows us to express the chern classes of $\eta$ in terms of those of $\gamma$. Furthermore it gives some relations among the chern classes of $\gamma$. Then one knows that the cohomology ring $H^{*}(G(m, n), Z)$ is the quotient of the polynomial ring $Z\left[c_{1}(\gamma), \cdots, c_{m}(\gamma)\right]$ by the ideal generated by the relations given by (2.1) (see Borel [1, p. 202]). Dually, $H_{*}(G(m, n), Z)$ is generated by the Schubert cycles (see Ehresmann [4]). Set $x=c_{1}\left(\gamma_{m}\right), y=c_{2}\left(\gamma_{m}\right) ; x$ and $y$ are dual, respectively, to Schubert cycles $X$ and $Y$, where $X$ is in fact nonsingular, as is $X^{2}$, the selfintersection of $X$.

We consider the problem: for which integers $a$ and $b$ can the cycle $a X^{2}+b Y$ be smoothed? One also has a stronger question: for which integers $a$ and $b$ is the cohomology class $\delta\left(a X^{2}+b Y\right)$ dual to a connected nonsingular variety?

As remarked above, the cycles $X$ and $X^{2}$ are given by nonsingular varieties; also, in $G(2,3), Y$ is a nonsingular variety (because it is dual to $c_{2}\left(\gamma_{2}\right)$ ). Thus on $G(2,3)$, every cycle $a X^{2}+b Y$ can be smoothed. Our example is on $G(3,3)$.

THEOREM 1. On $G(3,3)$ neither the cycle $Y$ nor the cycle $X^{2}+Y$ can be smoothed up to homological equivalence.

We in fact prove a stronger result: consider the dual cohomology class $a x^{2}+b y$ in $H^{4}(G(m, n) ; Z)$.

THeOREM 2. In $G(3,3)$ if $a x^{2}+b y$ is dual (mod 2) to a smooth submanifold, then $b$ is even. 
Note that this implies Theorem 1 , because every nonsingular subvariety is in particular a smooth submanifold, and any linear combination of cohomology classes with $b$ even still has $b$ even.

Proof of THEOREM 2. We use the work of Thom [11]: if $M$ is a smooth manifold and if $v \in H^{n}(M, Z / 2 Z)$, then $v$ is dual to a smooth submanifold of $M$ if, and only if, there is a map $f: M \rightarrow M O(n)$ such that $f^{*} U_{n}=v$, where $M O(n)$ is the Thom complex and $U_{n}$ the Thom class for the orthogonal group $O(n)$. Thus we prove Theorem 2 by showing that if there is a map $f: G(3,3) \rightarrow M O(4)$ with $f^{*} U=a x^{2}+b y$, then $b$ is even. (We set $U=U_{4}$.) The map $f$ induces a map $f^{*}: H^{*}(M O(4), Z / 2 Z) \rightarrow H^{*}(G(3,3), Z / 2 Z)$ which must be compatible with the Steenrod squaring operations. To carry out the proof, we need to know the cohomology of $M O(4)$ and the Steenrod operations, which are given in Thom [11]; the cohomology of $G(3,3)$, which can be computed from (2.1) above; and the Steenrod operations there, which are computed in Borel and Serre [3]. Here is our calculation: we have $\mathrm{Sq}^{2} U=U \cdot w_{2}$ and $\mathrm{Sq}^{2} y=x y+z$, where $z=c_{3}\left(\gamma_{3}\right)(\bmod 2)$. Thus, $f^{*}\left(U \cdot w_{2}\right)=b(x y+z)$. Suppose that $b$ is odd-we show this leads to a contradiction. Using the fact that $U \cdot\left(U \cdot w_{2}^{2}\right)=\left(U \cdot w_{2}\right)^{2}$, one deduces that $f^{*}\left(U \cdot w_{2}^{2}\right)=a x^{2} y+(1-a) y^{2}$. Now $\operatorname{Sq}^{2}\left(U \cdot w_{2}^{2}\right)=U \cdot w_{2}^{3}+U \cdot w_{3}^{2}$; but $f^{*}\left(U \cdot w_{3}^{2}\right)=0$ and $\mathrm{Sq}^{2} y^{2}=0$. Thus, $f^{*}\left(U \cdot w_{2}^{3}\right)=a\left(x^{3} y+x^{2} z\right)=0$, using (2.1), and consequently,

$$
f^{*}\left(U \cdot w_{2}^{2}\right)^{2}=f^{*}\left(U \cdot w_{2}\right) \cdot f^{*}\left(U \cdot w_{2}^{3}\right)=0 .
$$

This implies that

$$
\left(a x^{2} y+(1-a) y^{2}\right)^{2}=a x^{4} y^{2}+(1-a) y^{4}=0 .
$$

But by relation $2.1, x^{4} y^{2}=y^{4}$, and so we obtain $a+(1-a)=0(\bmod 2)$, which is impossible. Thus $b$ is even, which completes the proof of Theorem 2.

3. Further results. We now indicate without proof some further results which one can obtain by similar methods.

Again we consider the Grassmann variety $G(m, n)$. Suppose that the cycle $a X^{2}+b Y$ can be realized by a connected nonsingular variety. Such a variety is, in particular, a smooth submanifold with complex normal bundle.

THEOREM 3. In $G(2,3)$, the cohomology class by is dual to a smooth submanifold with complex normal bundle if and only if $b \equiv 0,1(\bmod 4)$.

Since there is a natural smooth embedding of $G(2,3)$ in $G(3,3)$, we can combine this result with Theorem 2 to obtain

Corollary. In $G(3,3)$, if by is dual to a smooth submanifold with complex normal bundle, then $b \equiv 0(\bmod 4)$. 
We in fact have a complete determination of which classes in $H^{4}(G(2,3)$, $\boldsymbol{Z})$ are dual to smooth submanifolds with complex normal bundle, but the statement of this result is too complicated to give here. It is a consequence of the following general result on manifolds of (real) dimension $\leqq 12$. (Note that $G(2,3)$ has dimension 12 .)

THEOREM 4. Let $M$ be a simply connected smooth manifold of dimension $\leqq 12$, and let $u \in H^{4}(M ; Z)$. Then $u$ is dual to a smooth submanifold with complex normal bundle if and only if, there are classes $v \in H^{6}(M ; Z)$ and $w \in H^{8}(M ; Z)$ such that

(i) $v(\bmod 2)=\mathrm{Sq}^{2} u$;

(ii) $w(\bmod 2) \in \Phi_{4}(u)$;

(iii) $w(\bmod 3)=\mathscr{P}_{3}^{1}(u)-u^{2}$;

(iv) $v^{2}=u w$.

Here $\Phi_{4}$ is a secondary operation associated with the unstable relation: $\mathrm{Sq}^{2} \mathrm{Sq}^{3}=0$, on integral classes of dimension 4 .

One proves Theorem 4 by again using Thom's theorem-thus one seeks a map $f: M \rightarrow M U(2)$, with $f^{*} U=u$. The fact that (i)-(iv) suffice to give the map $f$ is shown by constructing a Postnikov resolution of $M U(2)$, through $\operatorname{dim} 12$; the only difficulty comes in proving that $\pi_{11}(M U(2)) \cong Z$.

Given a cohomology class $y$ of even degree on a manifold $M$, Thom [11] shows that there is a nonzero integer $q$ such that the class $q y$ is dual to a submanifold with complex normal bundle. We remark that the set of such integers $q$ is always a union of cosets of a subgroup of the integers.

In particular, there is an integer $q \neq 0$ such that the class $q y \in H^{4}(G(3,3)$, $\boldsymbol{Z}$ ) is dual to a smooth submanifold (with complex normal bundle if we like). However, this is not a "stable" fact, as indicated by the following result: set $y_{m, n}=c_{2}\left(\gamma_{m}\right)$ in $H^{4}(G(m, n) ; Z)$.

THEOREM 5. For any integer $q$ there are integers $m, n$ such that in $H^{4}(G(m, n) ; Z), q y_{m, n}$ is not dual to a smooth submanifold.

The proof follows the same line as that given for Theorem 2, using the reduced $p$ th powers $\mathscr{P}_{p}^{i}$, and calculations by Borel-Serre [3].

4. Analogous results in characteristic $p>0$. Let $k$ be an algebraically closed field. Then we can consider the Grassmann variety $G(m, n)$ over $k$. It is a nonsingular projective variety over $k$, and it has Schubert cycles just as in the classical case.

THEOREM 6. Assume char $k \neq 2$. Then on $G(3,3)$ neither the cycle $Y$ nor the cycle $X^{2}+Y$ can be smoothed for algebraic or rational equivalence.

To prove this result we need a suitable cohomology theory in which Steenrod squares can be defined. Then we adapt the proof above. Epstein 
[5] has established a general theory of Steenrod cohomology operations, and Mme. Raynaud [10] has shown that Epstein's theory applies to the étale cohomology of schemes. In particular, the Steenrod squares are defined in $H_{e t}^{*}(G, Z / 2 Z)$ whenever $G$ is a scheme over a field of characteristic $p \neq 2$. Furthermore, since the Grassmann variety is defined over Spec $Z$, one of Mme. Raynaud's results shows that the cohomology ring of the Grassmann variety, together with the extra structure consisting of the Steenrod squares, is the same as the usual topological one.

Now suppose that $V$ is a nonsingular subvariety of $G=G(3,3)$ which is rationally (or algebraically) equivalent to $a X^{2}+b Y$. Then we show that $b$ is even, as in Theorem 2. We consider the inclusion $j: V \rightarrow G$. Then we can rephrase the proof of Theorem 2 above purely in terms of the cohomology rings of $V$ and $G$, and the maps $j^{*}, j_{*}$ between them. So we avoid mention of the Thom space $M O(4)$, and we can carry out the same proof.

In the case of characteristic $p=2$ this method does not work. However, it seems likely that one can obtain the same result in this case also, by using the Hodge cohomology $H^{i}(X)=\sum_{p+q=i} H^{q}\left(X, \Omega^{p}\right)$.

\section{REFERENCES}

1. A. Borel, Sur la cohomologie des espaces fibrés principaux et des espaces homogènes de groupes de Lie compacts, Ann. of Math. (2) 57 (1953), 115-207. MR 14, 490.

2. A. Borel and A. Haefliger, La classe d'homologie fondamentale d'un espace analytique, Bull. Soc. Math. France 89 (1961), 461-513. MR 26 \#6990.

3. A. Borel and J.-P. Serre, Groupes de Lie et puissances réduites de Steenrod, Amer. J. Math. 75 (1953), 409-448. MR 15, 338.

4. C. Ehresmann, Sur la topologie de certains espaces homogènes, Ann. of Math. 35 (1934), 396-443.

5. D. B. A. Epstein, Steenrod operations in homological algebra, Invent. Math. 1 (1966), 152-208. MR 33 \#7389.

6. H. Hironaka, Resolution of singularities of an algebraic variety over a field of characteristic zero. I, II, Ann. of Math. (2) 79 (1964), 109-326. MR 33 \#7333.

7. — Smoothing of algebraic cycles of small dimensions, Amer. J. Math. 90 (1968), 1-54. MR 37 \#210.

8. S. Kleiman, Geometry on Grassmannians and applications to splitting bundles and smoothing cycles, Inst. Hautes Études Sci. Publ. Math. No. 36 (1969), 281-297. MR 42 \#281.

9. S. Kleiman and J. Landolfi, Geometry and deformation of special Schubert varieties, Compositio Math. 23 (1971), 407-434.

10. M. Raynaud, Modules projectifs universels, Invent. Math. 6 (1968), 1-26.

11. R. Thom, Quelques propriétés globales des variétés différentiables, Comment. Math. Helv. 28 (1954), 17-86. MR 15, 890.

Department of Mathematics, University of California, Berkeley, California 94720

Mathematical Institute, Oxford, England OX1 3LB 